\title{
Laudes e cantigas espirituais de mestre André Dias: finalidade crítica e correlação com a obra de Jacoponi da Todi
}

\author{
Veridiana Skocic ${ }^{1}$ \\ Universidade do Estado do Rio de Janeiro \\ veridiana.skocic@gmail.com
}

\begin{abstract}
RESUMO: O presente artigo tem por objetivo demonstrar a perspectiva crítica da poesia religiosa medieval a partir de um breve estudo da obra intitulada Laudes e Cantigas Espirituais, escrita pelo beneditino André Dias na primeira metade do século XV, e sua correlação com a poesia religiosa italiana de Jacoponi da Todi. Reitera-se que o texto aqui apresentado é fruto de parte de um vasto estudo sobre as laudes de André Dias, cujas composições, em constante diálogo com os laudários italianos, que lhes antecederam, assumem, além de um valor pedagógico e edificante, também um escopo crítico em consonância com os escritos de Jacoponi.
\end{abstract}

Palavras-chave: Literatura religiosa. Laudes. Crítica. Idade Média.

ABSTRACT: The aim of this article is to show the critical perspective of medieval religious poetry through a brief study on Lauds and Spiritual Songs, written by the Benedictine André Dias in the first half of the $15^{\text {th }}$ century, and its correlation with the Italian religious poetry of Jacopone da Todi. We reinforce that the text presented here is the result of a comprehensive study on André Dias' lauds, in which the compositions, in a constant dialogue with the Italian laudaries that came before, assume, besides an edifying and pedagogical value, also critical scope in agreement with the writings of Jacopone.

Keywords: Lauds. Criticism. Middle Ages.

\section{Introdução}

Abordar o tema da poesia religiosa medieval implica evocar uma realidade teológica cuja essência se concentra no Novo Testamento, isto é, na Palavra de Deus encarnada em Jesus e documentada, sobretudo, nos Evangelhos e nos Atos dos Apóstolos.

\footnotetext{
${ }^{1}$ Doutora em Filologia portuguesa pela USP, mestre em Literatura italiana pela USP. Atualmente, é Professora Adjunta de Língua, Literatura e Cultura italianas na UERJ.
} 
Laudes e cantigas espirituais de mestre André Dias: finalidade crítica e correlação com a obra de Jacoponi da Todi

Veridiana Skocic

Assumindo, em muitos momentos, a função de estabelecer e de intensificar a relação entre Deus e o homem, a poesia religiosa pode ser considerada um instrumento importante pelo qual o crente é convidado à reflexão e, por conseguinte, à conversão e à redenção individual e/ou coletiva. Ao anunciar a Boa Nova, esse tipo de literatura passa a assumir também a função de exortar e ensinar. Observa-se, todavia, o caráter crítico dessas composições e é sob essa perspectiva que o presente artigo tem por objetivo apresentar ao leitor as Laudes e cantigas espirituais, escritas pelo beneditino André Dias de Escobar, religioso português do século XV, demonstrando o viés crítico de seus escritos e a correlação de sua obra com a poesia religiosa italiana medieval.

Ao entrarmos em contato com o laudário do beneditino, de pronto, a sensibilidade de suas páginas poéticas nos chamaram a atenção. À medida que avançávamos nas leituras, começávamos a perceber não só a finalidade pedagógica, doutrinária e crítica daqueles textos, mas também uma suposta relação com alguns escritos da literatura italiana medieval pela qual temos grande apreço. A suspeita de uma possível conexão da obra portuguesa com a literatura religiosa italiana fora, então, fomentada pelo fato de André Dias ter vivido em Florença e por ter sido ele o responsável pela difusão, em território português, da corrente espiritual do Santíssimo Nome de Jesus, cujo precursor havia sido o frade italiano Bernardino de Siena, seu contemporâneo. A partir dessa primeira percepção, passamos a pesquisar as obras de Feo Belcari e Jacopone da Todi cujas sacras representações e laudes ocupam um lugar de proeminência na literatura medieval da península itálica. Pudemos observar, então, que, assim como em Dias, os escritos desses autores também eram direcionados a um mesmo propósito: edificação, doutrina e crítica.

O diálogo entre a poesia religiosa medieval portuguesa e Laudes e Cantigas Espirituais. 
Laudes e cantigas espirituais de mestre André Dias: finalidade crítica e correlação com a obra de Jacoponi da Todi

Veridiana Skocic

É no seio das confraternidades medievais que o canto laudístico passa a assumir um papel de destaque enquanto manifestação devocional.

O desenvolvimento desse gênero de composição é verificado a partir da metade do século XIII com a difusão do movimento religioso de Raniero Fasani (? -1281) na Itália central. Fasani, um eremita franciscano, foi o precursor do movimento dos flagellanti ou disciplinati que anunciavam o advento da Idade do Espírito Santo, profetizada anos antes por Gioachino da Fiore.

A irmandade dos Disciplinati di Gesù Cristo, a exemplo de outras fraternidades, adotava a autoflagelação e a penitência como instrumentos de reconciliação com Deus. É nesse ambiente de extrema devoção, mortificação corporal e aspiração ascética que as procissões dos Flagellanti vinham acompanhadas por cantos de louvores a Deus e à Virgem.

Com o tempo, os cantos de louvor ou laudes, cantadas em língua vulgar pelo povo nas ruas, começaram a ser transcritas pelas Ordens Mendicantes nos chamados laudari ${ }^{2}$, espécie de cadernos nos quais eram escritas as composições destinadas à execução litúrgica.

A preferência pela língua vulgar é uma das características dessas composições destinadas, fundamentalmente, ao canto, e que privilegiavam, portanto, a tradição oral de caráter coletivo, assumindo, assim, um aspecto tipicamente popular.

A produção laudística assume um papel de relevância dentro da literatura italiana. Com efeito, é a laude Cantico delle creature, escrita em língua vulgar por Francisco de Assis, a obra inaugural da literatura italiana (CURTIUS, E. 1996, p.66). Classificada por Le Goff como a "mais bela obra de poesia religiosa desde os Evangelhos” (2001, p.101), destacam-se na composição não apenas a

\footnotetext{
2 Um dos mais importantes laudários é o Laudário de Cortona, coletânea de textos copiados entre 1270 e 1297 e que pertencia, nesse período, à Fraternidade de Santa Maria das Laudes da igreja de São Francisco de Cortona. Com notações musicais, é considerada a mais antiga coleção de música italiana em língua vulgar.
} 
Laudes e cantigas espirituais de mestre André Dias: finalidade crítica e correlação com a obra de Jacoponi da Todi

Veridiana Skocic

inovação lingüística, mas também o seu conteúdo ideológico que prega a importância de todas as criaturas de Deus, dentre as quais a figura central é o homem: o sol é o "irmão" que ilumina os dias do homem; o fogo ilumina a sua noite, e a terra produz flores e também frutos e ervas para o seu sustento. A título ilustrativo, transcrevemos abaixo alguns versos da laude do Santo de Assis:

A exemplo do Cantico di Frate sole ou dos sermões de Bernardino da Siena é possível afirmar que, do ponto de vista linguístico, Laudes e Cantigas Espirituais também se inserem num conjunto literário direcionado, essencialmente, ao povo. Como o próprio autor nos revela: "Laudas e cantigas spirituaes [...] trasladadas de lynguagem felorentyno em lynguagem portugaleso[...]", (DIAS, 1951, p. 17), ou seja, de língua vulgar para língua vulgar. É lícito supor, portanto, que não obstante as várias obras escritas em latim, o bispo português preferiu compor o seu laudário em língua vulgar não apenas para melhor expressar a sua espiritualidade e devoção, mas também para atribuir às suas laudes maior eficácia catequética, procurando transmitir de forma simples e acessível a mensagem das Escrituras.

Sob esta perspectiva, distinguem-se, ainda, as composições dramáticas cujo precursor fora Francisco de Assis. Ao organizar verdadeiras encenações quando pregava em praça pública ou quando montava presépios por ocasião do Natal (LE GOFF, 2001, p. 223), Francisco dá início ao desenvolvimento das representações religiosas. De fato, a encenação do nascimento do Menino Jesus idealizada pelo Santo e as laudes dramáticas podem ser consideradas os embriões das sacras representações do século XV, conforme atesta Angelina Visalli: "[...] apontamos para uma evolução desses textos no sentido teatral: no século XV as laudes ditas "dramáticas" se desdobram nas "sacras representações" (2004, p. 60). 
Laudes e cantigas espirituais de mestre André Dias: finalidade crítica e correlação com a obra de Jacoponi da Todi

Veridiana Skocic

De uma forma geral, pode-se observar que os temas sobre a Natividade, sobre a Paixão de Cristo e louvores (a Maria, a Jesus, aos santos) constituem a base das composições laudísticas. Note-se, entretanto, que estudiosos da literatura identificam dois grupos de laudes: líricas e dramáticas. As primeiras, via de regra, seriam destinadas aos louvores e ao nascimento de Cristo; as demais explorariam o sofrimento e morte de Jesus, as dores de Maria e os temas penitenciais.

No que tange ao laudário português, é possível verificar a presença de ambos os grupos. Notar-se-à ao longo das análises das composições que, de fato, as loas de Natal e os louvores a Nossa Senhora se caracterizam pelo tom acentuadamente lírico, traduzido, sobretudo, pelas figuras de linguagem (principalmente nos textos dedicados a Maria). Nas loas da Paixão e nos Prantos de Nossa Senhora, por outro lado, distinguem-se os diálogos entre Jesus e Maria ou Maria e o penitente.

Os temas são repetitivos, mas a recorrência temática é motivada pela proposta catequética, evangelizadora, doutrinal e devocional das composições que visam à consolidação da fé e ao ensinamento religioso. Com essa mesma finalidade, as encenações recorriam ao drama em movimento e aos elementos cênicos para captar maior atenção do público. Ratificando a função pedagógica das laudes, observe-se o caráter coletivo dessa forma de expressão devocional (não nos esqueçamos de que essas composições eram entoadas no seio das confraternidades). Neste sentido, podemos considerar a tradição laudística como propiciadora de coesão social:

Parece-nos que as confraternidades em geral, e o canto coletivo em particular, cumprem uma função dupla e de difícil separação, social e religiosa, lembrando que, para o homem do período, a religião não está separada de outras esferas da vida (VISALLI, 2004, p. 68).

O desenvolvimento do gênero laudístico é associado, particularmente, ao movimento franciscano, o qual contribuiu, de forma singular, para a difusão 
Laudes e cantigas espirituais de mestre André Dias: finalidade crítica e correlação com a obra de Jacoponi da Todi

Veridiana Skocic

dessas composições. De fato, o conteúdo das laudes demonstra a espiritualidade preconizada por Francisco, ou seja, uma religiosidade "horizontalizada", direcionada a homens, mulheres, ricos, pobres, sãos e doentes que buscam a mensagem do Evangelho a partir da contemplação dos ditos e feitos de Jesus e Maria.

Essa espiritualidade desenvolvida no âmbito das confrarias pode ser contemplada, em grande parte, através do conteúdo das laudes medievais, dentre as quais aquelas de André Dias. Em conformidade com suas antecessoras italianas, as composições portuguesas são orientadas pelo desejo de exortar, edificar, admoestar e exaltar. $\mathrm{O}$ valor doutrinário e evangelizador desse tipo de manifestação religiosa parece-nos evidente. Entretanto, é possível distinguir uma terceira finalidade nesses textos: a crítica.

\section{A laude como instrumento de crítica}

Ao estudarmos o laudário de André Dias, percebemos que uma composição se destaca das demais. Trata-se da laude Cantar que diram os desenparados e em este mundo atribulados, e seram per Jhesu livrados, em que é possível detectar a "faceta" crítica do autor ao censurar o estado de decadência no qual se encontra a Igreja e a sociedade de sua época.

Interessante observar a semelhança entre a composição portuguesa e a laude Del Pianto de la Chiesa redutta a mal stato, composta dois séculos antes pelo italiano Jacopone da Todi. A fim de demonstrar a função crítica da produção laudística, transcrevemos e analisamos os textos português e italiano, respectivamente:

Cantar que diram os desenparados e em este mundo atribulados, e seram per Jhesu livrados.

Planto muyto doorado,

faça todo o mundo coitado,

porque o veemos muyto afortunado,

e posto em tribulaçom e muyto maoo estado.

E eu mym, e honde som os prophetas plenos de sabedoria, $\quad 5$ 


\section{Laudes e cantigas espirituais de mestre André Dias: finalidade crítica e correlação \\ com a obra de Jacoponi da Todi \\ Veridiana Skocic}

todo o mundo ora hecheo de mentira e de folia,

e nenguem nom quer ouvyr a verdade,

mays de boamente acha toda falsydade,

E engana a seu amygo com plazentearia,

Por que seja atribulado.

E hu som os patriarcas, plenos de fé e de creença, porque ja em todo o mundo, grandes e pequenos, Poserom e pooem toda sua vyda e femença, de guaançar senhorios e riqueza, e de cobiça e maleza,

todo o mundo he muyto fortemente mazelado.

E hu som os apostolos, preegadores da verdade, Jahy nomha nem huum atam ousado, de a preegar e dizer, mays assy huuns como os outros juntamente, se trabalham de preegar e dizer muyto altamente, mentiras e falssidades,

e assy enganam toda gente,

e nomhe achado soomente huum

que por o senhor Deus Jhesu se trabalhe

de fazer e obrar as obras de piedade,

em este mundo malfadado.

E hu som os martires plenos de fortaleza,

Ja os reys e príncipes e frades

cheos som todos de muyto grande fraqueza

de exalçar e defender a sancta fe christãa,

e padecer e morrer por ella e a averem de poer

em muyto alta e grande nobreza.

Ja o amor da sancta fe do senhor Deus Jhesu todo he anychilado.

E hu som os confessores,

Clerigos, religiosos e pastores,

que en no serviço de Deus Jhesu eram muyto ferventes

e por os seus boons exemplos e castigos

eram devotas todas as gentes,

de todo ora som muyto faleçentes,

e porem todo ho mundo he muyto atribulado.

$\mathrm{E}$ hu som os doutores plenos de sabedoria,

todos os vejo sayr de toda boa vyda,

e nom curam de sçiençia, nem de outra boa doutrina,

mays todos trabalham por averem ofícios,

e honras e benefícios e muytapecunya,

e pera ajuntarem em riqueza e muyto algo.

E hu som as virgens de alma e de vontade, 
e hu som os casados que mantenham

a hordem do casamento com toda lealdade

e hu som as vyuvas e as freiras professas

quem manteem auqella castidade

que per a sancta ley he estabelecido e mandado.

E hu som os religiosos e hyrmitãaes,

que vyvam em grande temperamento

Ja todos som lançados

em muy grande perdimento

priores e abades e monges

com todo seu convento,

todos de ssydamm aao exemplo,

e fazem a todos grande escandalo,

a todos muyto pubricado.

Oo amara e triste paz e folgança,

tu lançaste todo o mundo em muyto grande perdiçom,

por que enquanto durou o trabalho da egreja e da terra,

e se fazia por a fe[ de ] Christo guerra,

esteve todo o mundo, em muyto grande devaçom,

e agora no muyto grande asossego,

o diabo tem o poboo de Christo

em seu poder muytosojugado.

Partindo de uma análise preliminar da laude portuguesa, pode-se verificar que a composição revela a indignação do narrador quanto à tibieza e o estado de decadência não só da Igreja, mas da sociedade em geral. É por meio de uma série de perguntas retóricas que o narrador lança sua crítica às instituições religiosas e à sociedade, chamando a atenção para a corrupção dos costumes e para a avidez material do clero. Além da indignação, evidencia-se na primeira estrofe a tristeza e a desolação perante um cenário que reflete uma sociedade degradada espiritual e moralmente: “...mundo coytado...posto em tribulaçom e maoo estado”. A partir da segunda estrofe, até a sexta, o narrador se limita ao contexto religioso: falsos profetas, patriarcas, pregadores, frades, clérigos, pastores, enfim, todos são objeto de crítica. Nos versos 17-27, o narrador centraliza a crítica aos pregadores que "trabalham de preegar e dizer muyto altamente mentiras e falssidades, assy enganam toda gente". $\mathrm{Na}$ estrofe 
Laudes e cantigas espirituais de mestre André Dias: finalidade crítica e correlação com a obra de Jacoponi da Todi

Veridiana Skocic

seguinte, o objeto de crítica são as ordens mendicantes e a nobreza, outrora tão engajados em defender o cristianismo e a fé católica nas Cruzadas. A sétima estrofe ocupa-se dos homens do saber, os "doutores plenos de sabedoria”, que ávidos por poder e riquezas não mais se dedicam à atividade doutrinária, "outra boa doutrina”. Na oitava estrofe, a crítica recai sobre leigos e religiosos, destacando-se a figura feminina cujo comportamento pervertido depõe contra a preservação da castidade e da fidelidade. Em seguida, o alvo são as ordens monásticas; abades e monges que "som lançados em muy grande perdimento" graças, principalmente, às comendas "com todo seu convento, todos de ssy dam maao exemplo”. Finalmente, o narrador retoma o sentimento de amargura presente no início da composição, apontando a tibieza religiosa como a maior culpada pelo estado de degradação moral e espiritual da Igreja e da sociedade; enquanto religiosos e fiéis estiverem distantes de Deus, "o diabo tem o poboo de Christo em seu poder muyto sojugado.”

Passemos, a seguir, ao exame da composição Del Pianto de la Chiesa redutta a mal stato de Jacopone da Todi. Nesta laude, o franciscano põe em ena a Santa Madre Igreja lamentando a corrupção de seus filhos:

Piagne la Ecclesia, piagne e dolura: senza fortuna di pessimo stato.

O nobilissimamadre, che piangi? Mustre Che senti dolur molto magni: narrame 'l modo perché tanto lagni,

che sì duro pianto fai esmesurato?

Figlio, eo sì piango, ché m’ aio anvito:

veiome morto pate e marito;

figli, frategli, neputi ho smarrito, onne mio amico è preso e legato.

So circundata da figli bastardi:

Em omne mia pugna se mustra codardi;

Li miei legitimi, spade né dardi, lo lor coraio non era mutato. 
Li miei legitimi era'n concorda:

veio i bastardi pin de discórdia;

la gente enfedele me chiama la lorda

per lo rio essempio che ò semenato.

Veio esbannita la povertate

nullo è che cure si no 'n degnetate;

li miei legitimi em asperetate,

tutto lo monno ro fo conculcato.

Auroed argento ò rebannito, fatt'ònemici con lor gran convito, omne buon uso da loro è fugito:

donne 'l mio pianto con granneeiulato.

O'so li? Patripieni di fide

Null'è che cure morir l'om me vide:

la tepedezam'ha preso edoccide, el miodolore non è corrottato.

O' so i profeti pin de speranza?

Null'è che cure en miavedovanza;

Presonzione presa ha baldanza,

Tutto lo monno po' lei s'è rizato.

O' so l' appostoli pin de fervore?

Null' è he cure en mio dolore:

Escito m' è scontra lo proprio amore,

e ia non veio che i sia contrastato.

O' so gli martiri pin de forteza?

Non è che cure em mia vedoveza:

escita m' è scontra l' ageveleza,

el mio fervore sì ha nichilato.

O' so i prelati iusti e fervente,

che la lor vita sanava la gente

Escit'è la pompa, grossura potente,

E sì Nobel ordene m' ha maculato.

O' so i dotturi pin de prudenza?

Multi ne veio saliti en escienza; ma la lor vita non m'ha convegnenza: dato m'òcalci che 'l cor m' ò accorato.

O reliusi em temperamento, granne de vui avea piacemento; orvo cercano onne convento, pochine trovo en cui sia consolato. 
Mentre fui em pugna, sì stetti dritta; or lo reposo m'ha presa e sconfitta, el blando dracone sì m' ha venenato.

Null' è che venga al mio corrotto, en ciascun statosì m' è Cristo morto: o vita mia, speranza e deporto, em onne coraio te veio affocato.

Assim, como a laude portuguesa, também esta composição trata da corrupção da Igreja e da tibieza de sacerdotes e da sociedade.

Não obstante a conformidade entre os temas, é possível distinguir a cosmovisão de cada autor. Com efeito, ao cotejarmos a composição portuguesa com o texto iacopônico, conseguimos notar "atitudes" diferenciadas perante uma mesma temática - a degradação da sociedade e da instituição religiosa. Neste sentido, André Dias adota o texto italiano como referência para a sua composição, assumindo-o como base e, a partir dele, agregando ou suprimindo elementos de acordo com sua visão de mundo. Mário Martins observa:

[...] as versões de mestre André Dias desprendem-se do original, ganham o valor duma criação poética que nos para levantar vôo[...]. Mesmo quando se não liberta tanto da lauda toscana, podemos afirmar que Mestre André, mais de que trasladar para lynguagem portugaleso, regia portugalesmente, se nos permitem o advérbio, e criava, sobre o mesmo tema musical e ideológico, uma poesia nova[...].lembra, aqui e ali, os nossos versos modernistas. Os versos italianos, neste caso, serviam de ponto de apoio (MARTINS, 1951, pp .25-26).

A afirmação do estudioso atesta, pois, a relação das laudes portuguesas com os escritos de Jacopone. Entretanto, conforme podemos observar, esse diálogo se dá, sobretudo, pela reelaboração do conteúdo original a partir do qual é construída uma nova poesia. André Dias adapta, assim, o texto italiano de acordo com as exigências de seu espírito reformador, ávido por criticar não apenas o estado da Igreja, mas também o laicado.

No que tange às duas composições analisadas, as semelhanças aparecem a partir da sétima estrofe, uma vez que Mestre André "descarta" as primeiras 
Laudes e cantigas espirituais de mestre André Dias: finalidade crítica e correlação com a obra de Jacoponi da Todi

Veridiana Skocic

estâncias da composição italiana. São os versos iniciais de cada estrofe subsequente que demonstram a correspondência entre os textos ou até a tradução literal da composição de Jacopone pelo bispo de Mégara:

Ó so li patri pieni di fede?

O' so i profeti pin de speranza?

O' so gli appostoli pin de fervore?

O' so gli martiri pin de forteza?

O' su i dotturi pin de prudenza?

O pace amara, com'hai afflitta!

Mentre fui em pugna,

si stetti dritta; or lo reposo m'ha presa e sconfitta, el brando dracone si m'ha venenato
E hu som os patriarchas plenos de fé e de creença e honde som os prophetas plenos de sabedoria $\mathrm{E}$ hu som os apóstolos, preegadores da verdade E hu som os mártires plenos de fortaleza

$\mathrm{E}$ hu soom os doutores plenos de sabedoria

Oo amara e triste paz e folgança...porquanto durou o trabalho da egreja e da terra...esteve todo mundo, em muyto grande devoçom, e agora no muyto grande asossego, o diabo tem o poboo de Christo.

Ainda que haja cá e lá trechos traduzidos ipsis litteris, André Dias adota o texto iacopônico apenas como base para sua composição, reformulando o conteúdo original, invertendo a ordem de estrofes e versos. Sua crítica é mais abrangente: para o bispo português ninguém se salva. Todos, leigos e religiosos, estão corrompidos, e a inclusão deste novo elemento, isto é, o leigo, constitui um fator de destaque com relação ao texto italiano. Enquanto Jacopone se restringe apenas à imoralidade dos religiosos: "So circundata da figli bastardi”, Dias critica não só a decadência no seio da Igreja, mas também os vícios da sociedade (oitava estrofe).

Destaca-se que o bispo de Mégara, além de inserir elementos novos no plano do conteúdo, também reconfigura estruturalmente a composição original, invertendo a ordem das estrofes e mudando o foco narrativo. $\mathrm{Na}$ composição portuguesa, o narrador em $1^{\text {a }}$ pessoa lança uma série de perguntas, sem obter as respostas. Na laude italiana, por outro lado, essa relação se estabelece, primeiramente, em forma de diálogo $1^{\text {a }}$ pessoa/interlocutor. Lançando mão do recurso da personificação, o sujeito poético se dirige à Igreja: "O nobelissima mamma, che piangi?”. A partir da 
Laudes e cantigas espirituais de mestre André Dias: finalidade crítica e correlação com a obra de Jacoponi da Todi

Veridiana Skocic

segunda estrofe, até o final, há uma mudança de foco narrativo, pois é a Igreja quem se lamenta e quem lança as perguntas, passando, assim, de interlocutor a narrador em $1^{a}$ pessoa. Observe-se que em ambas as composições a ausência de respostas demonstra a desesperança e o pessimismo dos autores.

\section{Considerações finais}

Conclui-se, por fim, que tanto Jacopone quanto André Dias fazem críticas severas aos costumes decadentes da Igreja. Suas laudes testemunham a indignação contra a corrupção dos religiosos e, no caso de Dias, também contra a ruína moral que assola a sociedade de seu tempo. O franciscano, vivenciando um processo no qual o clero graças à riqueza e ao conhecimento que tinha em mãos tornou-se ambicioso e corrompido, faz uso da personificação (em sintonia com o alegorismo medieval) através da qual a Igreja lamenta a corrupção de seus membros, demonstrando indignação perante a decadência da Cúria Romana; o bispo português, testemunhando um momento crucial desse estado de degradação que atinge também a vida secular, assume como referência o texto italiano, reconstruindo-o a partir de sua vivência, seu ambiente e de suas percepções.

A convergência entre as laudes do bispo de Megara e os escritos do franciscano Jacopone da Todi reside na crítica à tibieza e à degradação moral da Igreja e da sociedade. Dias e Jacopone atribuem uma função crítica, além daquela catequética, às laudes, conforme pudemos observar nas composições Del pianto de la chiesa redutta a mal stato do frade italiano e Cantar que diram os desenparados. Desta forma, além da intenção didática, esse tipo de manifestação religiosa parece ter sido também um eficaz instrumento de censura.

Por fim, diante do exposto, é possível inferir que a laude (enquanto parte integrante da literatura religiosa), não obstante ter como base um quadro temático menos vasto e uma proposta mais específica que a da literatura laica, 
Laudes e cantigas espirituais de mestre André Dias: finalidade crítica e correlação com a obra de Jacoponi da Todi

Veridiana Skocic

é igualmente diversificada na medida em que seus autores, ainda que visem a uma finalidade comum e estejam limitados à recorrência temática, conseguem realizá-la de formas distintas a partir de sua mundividência.

\section{Referêcias bibliográficas}

ALMEIDA Fortunato. História da Igreja em Portugal. Nova Edição, 4 vols. Porto: Livraria Civilização Editora, 1967-1971.

CALDERÓN, Manuel. O Livro de orações em prosa e verso vulgar com as laudas e cãtigas spirituaes de André Dias. In: Signum. Revista da Associação Brasileira de Estudos Medievais, 6, 2004, pp.45-88.

CANETTIERI, Paolo. “Laude di Jacopone da Todi.”In: Letteratura Italiana dalle origini al Cinquecento. Torino: Einaudi, 1992.

DE SANCTIS, Francesco. Historia della literatura italiana. Buenos Aires: Editorial América, s/d, pp.32-33.

DIAS, André. Laudes e cantigas espirituais. Coligidas, anotadas e comentadas por Mário Martins. Lisboa: Oficinas Ramos, Afonso \& Moita, 1951.

GRENDI, Edoardo. "Leconfraternitecomefenomenoassociativoereligioso", In Società, Chiesa e vita religiosa nell" "ancientrégime', a cura di C. Russo. Napoli, 1976.

MARQUES, Antonio Henrique. A Sociedade Medieval Portuguesa. 5a. Ed. Lisboa: Livraria Sá da Costa, 1987.

MARTINS, Mario. A Bíblia na literatura medieval portuguesa. $1^{\text {a }}$ ed. Lisboa: Livraria Bertrand Ed., 1979.

RICCI, Angelo. Umanità e popolo nella lauda del secolo XIII. Porto Alegre: Imprensa Universitária Editora, 1971.

ROMAG, Dagoberto. Compêndio de história da Igreja - Idade Média. Vol.II. $2^{\mathrm{a}}$ ed. Petrópolis: Vozes, 1950.

TODI, Jacoponi. Le laudi. A cura di Giovanni Ferri. Laterza, 1915. 
Laudes e cantigas espirituais de mestre André Dias: finalidade crítica e correlação com a obra de Jacoponi da Todi

Veridiana Skocic

VISALLI, Angelita. Cantando até que a morte nos salve: estudo sobre as laudas italianas dos séculos XIII e XIV. Tese de doutorado, USP, São Paulo, 2004. 


\section{Laude e canzoni spirituali del maestro André Dias: finalità critica e collegamento con l'opera di Jacopone da Todi ${ }^{3}$}

Veridiana Skocic ${ }^{4}$

RIASSUNTO: Il presente articolo ha come obbiettivo quello didimostrare la prospettiva critica della poesia religiosa medievale a partire da un breve studio dell'opera intitolata Laudes e Cantigas Espirituais scritta dal fratebenedettino André Dias nella prima metà del XV secolo e, il suo collegamento con la poesia religiosa italiana di Jacoponi da Todi. È giusto ricordare che il testo qui presentato fa parte di un vasto studio sulle laudi di André Dias i cui componimenti, in costante dialogo con le precedenti poesie religiose italiane, assumono, oltre ad un valore educativo ed edificanteper lo spirito, anche una portata critica in linea con gli scritti di Jacopone.

Parole-chiave: Lodi. Critica. Portogallo. Italia. Medioevo.

ABSTRACT: The aimofthisarticleis to show the critical perspective of medieval religious poetry through a brief study on Lauds and Spiritual Songs, written by the Benedictine André Dias in the first half of the $15^{\text {th }}$ century, and its correlation with the Italian religious poetry of Jacopone da Todi. We reinforce that the text presented here is the result of a comprehensive study on André Dias' lauds, in which the compositions, in a constant dialogue with the Italian laudaries that came before, assume, besides an edifying and pedagogical value, also critical scope in agreement with the writings of Jacopone.

Keywords: Lauds. Criticism. Middle Ages.

\section{Introduzione}

Affrontare la questione della poesia medievale religiosa implica evocare una realtà teologica la cui essenza viene concentrata nel Nuovo Testamento, cioè, nella parola di Dio incarnata in Gesù e documentata soprattutto nei Vangeli e negli Atti degli Apostoli.

\footnotetext{
3 Versione di Alcebiades Arêas, Edvaldo Sampaio Belizário e Teresa Cristina do Nascimento Jardin ${ }^{4}$ Dottoressa in Filologia Portoghese (USP). Attualmente, è docente di Lingua e Letteratura italiane presso la UERJ.
} 
Laudes e cantigas espirituais de mestre André Dias: finalidade crítica e correlação com a obra de Jacoponi da Todi

Veridiana Skocic

Assumendo, in molti momenti, la funzionedi stabilire ed intensificare le relazioni tra Dio e l'uomo, la poesia religiosa può essere considerata un importante strumento mediante il quale il credente è portato alla riflessione e, di conseguenza, alla conversione e alla redenzione individuale e/o collettiva. Nell'annunciare la Buona Novella, questo tipo di letteraturaassume il ruolo di esortare e di insegnare.

Si osserva, tuttavia, la natura critica di queste composizioni ed è in quest'ottica che il presente saggio vuolepresentare al lettore le Lodi e $i$ cantici spirituali, scritti dal frate benedettino André Dias de Escobar, religioso Portoghese del XV secolo, dimostrando l'aspetto critico dei suoi scritti e la sua correlazione con la poesia italiana religiosa medievale.

Quando entriamo in contatto con il Graduale Romanodei frati Benedettini, improvvisamente la sensibilitàdi questepagine poetiche ci colpisce. Man mano che scopriamo nuove letture, cominciamo a capire non solo lo scopo educativo, dottrinarioe critico di questi testi, ma anche una possibile relazione con alcuni scritti della letteratura medievale italiana dei quali abbiamo grande apprezzamento. Il sospetto di un possibilecollegamento tra le opere portoghesi con laletteratura religiosa italiana nasce dal fatto che André Dias ha vissuto a Firenze per poi essere responsabile della diffusione, nel territorio portoghese, della corrente spirituale del Santissimo nome di Gesù il cui precursore era il frate italiano Bernardino da Siena, suo contemporaneo. A partire da questo primo studio passiamo a conoscere le opere di FeoBelcari e di Jacopone da Todi le cui rappresentazioni e Lodi Sacre occupano un posto di rilievo nella letteratura medievale della penisola italiana. Possiamo osservare quindi che quanto succede con Dias, avviene anche con gli scritti di questi autori i quali sono diretti ad uno stesso proposito: l'edificazione umana, la dottrina e la critica.

Il dialogo tra la poesia religiosa medievale portoghese, le Lodi e i Cantici Spirituali 
Laudes e cantigas espirituais de mestre André Dias: finalidade crítica e correlação com a obra de Jacoponi da Todi

Veridiana Skocic

È all'interno delle confraternite medievali che il canto laudistico assume un ruolo di primo piano durante la manifestazione religiosa.

Lo sviluppo di questo genere di composizione avviene a partiredalla metà del XIII secolo con la diffusione del movimento religioso di Raniero Fasani (?-1281) in Italia centrale.Fasani, un eremita francescano, fu il precursore del movimento dei flagellanti o disciplinati che annunciano l'avvento dell'Età dello Spirito Santo, profetizzato anni prima da Gioacchino da Fiore.

La Confraternità dei Disciplinati di Gesù Cristo, come altri movimenti cattolici dell'epoca, adottava l'autoflagellazione e la penitenza come strumenti di riconciliazione con Dio.È in questo ambiente di estrema devozione, mortificazione e aspirazione ascetica che le processioni deiFlagellantivenivano accompagnate da canti di lode a Dio e alla Vergine.

Nel corso degli anni, i canti di lode o le Laudi, eseguite in volgare dal popolo per le strade, hanno cominciato ad essere trascritti dagli Ordini Mendicanti nel cosiddetto laudari $^{5}$, specie di quaderno su cui erano scritte le composizioni per le funzioni liturgiche.

La preferenza di un linguaggio volgare è una delle caratteristiche di questi componimenti destinati, fondamentalmente, al canto e che privilegiavanola tradizione orale di carattere collettivo, assumendo così un aspetto tipicamente popolare.

La produzione laudistica svolge un ruolo importante all'interno della letteratura italiana. Infatti, è la laude: Cantico delle creature, scritta in volgare da Francesco d'Assisi, l'opera inaugurale della letteratura italiana (CURTIUS, E. 1996, p. 66). Classificata da Le Goff come il "lavoro più bello di poesia religiosa dopo i Vangeli" (2001, p. 101). Nella composizione risalta non solo l'innovazione linguistica, ma anche il contenuto ideologico che predica

\footnotetext{
${ }^{5}$ Uno dei principal laudari è il Laudario di Cortona, collezione di laude copiate fra gli anni 1270 e 1297 e che apparteneva, in questo periodo, alla Fraternità di Santa Maria delle Laude della chiesa di San Francesco di Cortona. Il manoscritto con notazioni musicali è considerato la più antica collezione di musica italiana in lingua volgare.
} 
Laudes e cantigas espirituais de mestre André Dias: finalidade crítica e correlação com a obra de Jacoponi da Todi

Veridiana Skocic

l'importanza di tutte le Creature di Dio, dove la figura centrale è l'uomo: il sole è il "fratello" che illumina il giorno dell'uomo; il fuoco illumina la notte e la terra produce fiori e frutti ed erbe per il suo sostentamento. Per illustrare, trascriviamo qui di seguito alcuni versi dellalaudedel Santo di Assisi:

Con l'esempio del Cantico di Frate sole o le prediche di San Bernardino da Siena è possibile affermare che dal punto di vista linguistico, anche le Lodi e cantici spirituali si inserisconoin un insieme letterario indirizzato, essenzialmente, al popolo. Come lo stesso autore rivela: 'Laudas e cantigas spirituaes[...] trasladadas de lynguagem felorentyno em lynguagem portugaleso[...]" (MARTINS, 1951, p. 17), ossia, dalla lingua volgare alla lingua volgare. Dobbiamo pensarequindi che, nonostante le molte opere scritte in latino, il vescovo portoghese abbia scelto di comporre il laudario in lingua volgare non solo per meglio esprimere la sua spiritualità e devozione ma anche per dare maggiore efficacia catechistica cercando di trasmettere il messaggio delle Scritture in modo semplice e accessibile.

Sotto questo punto di vista c'è l'esempio di Francesco d'Assisi e delle sue composizioni drammatiche di cui lui fu il precursore. Nell'organizzare vere rappresentazioni quando predicava in pubblico o quando costruiva i presepi in occasione del Natale (LE GOFF, 2001, p. 223), Francesco diede inizio allo svolgimento delle sacre rappresentazioni. Infatti, sia l'atto di nascita del bambino Gesù ideato dal Santo, che le lodi drammatiche possono essere considerati veri embrioni delle sacre rappresentazioni del secolo XV come Angelina Visalli attesta: “[...] puntiamo ad un'evoluzione di questi testi nel senso teatrale: nel XV secolo le Lodi dette "drammatiche" sisvolgono nelle "sacre rappresentazioni" (2004, p. 60).

In generale, si può osservare che gli argomenti sulla Natività, la Passione di Cristo e le lodi (a Maria, aGesù, ai santi) sono la base delle composizioni laudistiche. Si osserva, tuttavia, che gli studiosi di letteratura identificano due gruppi di Lodi: liriche e drammatiche. Le prime, come regola generale, 
Laudes e cantigas espirituais de mestre André Dias: finalidade crítica e correlação com a obra de Jacoponi da Todi

Veridiana Skocic

sarebbero destinate alle lodi e alla nascita di Cristo; le altre invece esalterebbero la sofferenza e la morte di Gesù, il dolore di Maria e i temi penitenziali.

Per quanto riguarda il laudario portoghese, è possibile verificare la presenza di entrambi i gruppi. Attraverso l'analisi delle composizioni come le preghiere di Natale e le Lodi alla Madonna si nota come di fatto sono caratterizzati dal tono altamente lirico ispirato soprattutto dalleproprie figure (soprattutto nei testi dedicati a Maria). Nella Lode della Passione e nel Pianto della Madonna, d'altra parte, si distinguono i dialoghi tra Gesù e Maria o Maria e il penitente.

I temi sono ripetitivi, ma la ricorrenza tematica è motivata dalla proposta catechistica, evangelizzante, dottrinale e devozionale delle composizioni che mirano a consolidare la fede e l'insegnamento religioso. Con questo stesso scopo, le rappresentazioni utilizzavano il dramma in movimento e gli elementi scenici per attirare maggiore attenzione del pubblico. Convalidando la funzione pedagogica delle Lodi, si nota il carattere collettivo di questa forma di espressione devozionale (non dimentichiamo chequeste composizioni sono state cantate all'interno delle Comunità Religiose). In questo senso, possiamo considerare la tradizione laudistica come un fattore di coesione sociale:

Ci sembra che le Comunità Religiose in generale, e il canto collettivo in particolare, abbiano una doppia funzione e di difficile separazione religiosa e sociale, ricordando che, perl'uomodi quel periodo, la religione non era separata dalle altre situazioni della vita. (VISALLI, 2004, p. 68).

Lo sviluppo del genere laudistico è associato, in particolare, al movimento francescano che ha contribuito, in un modo unico alla diffusione di queste composizioni. Infatti, il contenuto della Lode dimostra la spiritualità idealizzata da Francesco, vale a dire, una religiosità "orizzontale", direzionata a uomini, a donne, a ricchi, a poveri, a sani e a malati che cercano il messaggio 
Laudes e cantigas espirituais de mestre André Dias: finalidade crítica e correlação com a obra de Jacoponi da Todi

Veridiana Skocic

del Vangelo a partire dalla contemplazione delle parole e delle azioni di Gesù e Maria.

Questa spiritualità sviluppata nell'ambito delle confraternite può essere contemplata, in gran parte, attraverso il contenuto delle Lodi medievali tra cui quelle di André Dias. Seguendo le antecedenti italiane, le composizioni portoghesi sono guidate dal desiderio di esortare, edificare, ammonire ed esaltare.Il valore dottrinale ed evangelizzatore di questo tipo di manifestazione religiosa sembra evidente.Tuttavia, è possibile distinguere un terzo scopo in questi testi: la critica.

\section{La laude come strumento di critica}

Studiando il laudario di André Dias, ci siamo resi conto che una delle composizioni si distingue dalle altre. Questa è la lode: Cantar che quediram os desenparados e em este mundo atribulados, e seram per Jhesu livrados dove è possibile osservare il carattere criticodell'autore quando lui censura lo stato di degrado in cui si trovano la Chiesa e la società di quel tempo.

È interessante osservare la somiglianza tra la composizione portoghese e la lode Del Pianto della Chiesa redutta a mal stato composta due secoli prima dall'italiano Jacopone da Todi. Al fine di dimostrare il ruolo critico della produzione laudistica, trascriviamo e analizziamo $i$ testi in portoghese e in italiano, rispettivamente:

\section{Cantar que diram os desenparados e em este mundo atribulados, e seram per Jhesu livrados}

Planto muyto doorado, faça todo o mundo coitado, porque o veemos muyto afortunado, e posto em tribulaçom e muytomaoo estado.

E eu mym, e honde som os prophetas plenos de sabedoria, $\quad 5$ todo o mundo ora hecheo de mentira e de folia, e nenguemnom quer ouvyr a verdade, mays de boamente acha toda falsydade, 


\section{Laudes e cantigas espirituais de mestre André Dias: finalidade crítica e correlação \\ com a obra de Jacoponi da Todi \\ Veridiana Skocic}

E engana a seu amygo com plazentearia, Por que seja atribulado.

E hu som os patriarcas, plenos de fé e de creença, porque ja em todo o mundo, grandes e pequenos, Poserom e pooem toda sua vyda e femença, de guaançar senhorios e riqueza, e de cobiça e maleza,

todo o mundo hemuyto fortemente mazelado.

E hu som os apostolos, preegadores da verdade, Jahynomha nem huum atam ousado, de a preegar e dizer, maysassyhuuns como os outros juntamente, se trabalham de preegar e dizer muyto altamente, mentiras e falssidades,

e assy enganam toda gente, e nomhe achado soomentehuum que por o senhor Deus Jhesu se trabalhe de fazer e obrar as obras de piedade, em este mundo malfadado.

E hu som os martires plenos de fortaleza, Ja os reys e príncipes e frades Cheos som todos de muyto grande fraqueza de exalçar e defender a sanctafechristãa, e padecer e morrer por ella e a averem de poer em muyto alta e grande nobreza.

Ja o amor da sanctafe do senhor Deus Jhesu todo heanychilado.

E hu som os confessores,

Clerigos, religiosos e pastores, que en no serviço de Deus Jhesu eram muyto ferventes

e por os seus boons exemplos e castigos eram devotas todas as gentes, de todo ora som muytofaleçentes, e porem todo ho mundo hemuyto atribulado.

E hu som os doutores plenos de sabedoria, todos os vejo sayr de toda boa vyda, e nom curam de sçiençia, nem de outra boa doutrina, mays todos trabalham por averemofícios,

e honras e benefícios e muytapecunya, e pera ajuntarem em riqueza e muyto algo.

E hu som as virgens de alma e de vontade, e hu som os casados que mantenham 
a hordem do casamento com toda lealdade

e hu som as vyuvas e as freiras professas

quem manteemauqella castidade

que per a sanctaleyhe estabelecido e mandado.

E hu som os religiosos e hyrmitãaes,

que vyvam em grande temperamento

Ja todos som lançados

em muy grande perdimento

priores e abades e monges

com todo seu convento,

todos de ssydammaaoexemplo,

e fazem a todos grande escandalo,

a todos muytopubricado.

Oo amara e triste paz e folgança,

tu lançaste todo o mundo em muyto grande perdiçom,

por que enquanto durou o trabalho da egreja e da terra,

e se fazia por a $\mathrm{fe}[\mathrm{de}]$ Christo guerra,

esteve todo o mundo, em muyto grande devaçom,

e agora no muyto grande asossego,

o diabo tem o poboo de Christo

em seu poder muytosojugado.

Partendo da un'analisi preliminare della lode portoghese, si può vedere che la composizione rivela l'indignazione del narratore circa la tiepidezza e lo stato di degrado non solo della Chiesa, ma della società in generale. È attraverso una serie di domande retoriche che il narratore lancia la sua critica alle istituzioni religiose e alla società, richiamando l'attenzione sulla corruzione dei costumi e l'avidità materiale del clero. Oltre all'indignazione, si trova nella prima strofa, la tristezza e la desolazione davanti a uno scenario che riflette una società degradata moralmente espiritualmente: “... mondo coytado... posto emtribulaçom e maoo estado". Dalla seconda alla sesta strofa il narratore si limita al contesto religioso: falsi profeti, patriarchi, predicatori, monaci, sacerdoti, pastori, comunque, tutti sono oggetto di critica. Nei versi 17-27 il narratore centralizza la critica ai predicatori che "trabalham de preegar e dizer muyto altamente mentiras e falssidades, assy enganam toda a gente". Nella 
Laudes e cantigas espirituais de mestre André Dias: finalidade crítica e correlação com a obra de Jacoponi da Todi

Veridiana Skocic

strofa seguente l'oggetto della critica sono gli ordini mendicanti e la nobiltà, una volta così impegnati a difendere il cristianesimo e la fede cattolica nelle crociate. La settima strofa si occupa degli uomini di sapere, i "dottori pieni di saggezza" che desiderosi di potere e ricchezze non si impegnano più nell'attività dottrinale "outra boa doutrina". Nell'ottava strofa la critica è per i laici e i religiosi, soprattutto per la figura femminile il cui comportamento pervertito agisce contro la preservazione della castità e fedeltà. In seguito l'obiettivo sono gli ordini monastici; abati e monaci che "som lançados em muy grande perdimento" dovuto, principalmente, per la lode "com todo seu convento, todos de ssy dam maao exemplo". Infine, il narratore riprende la sensazione di amarezza presente all'inizio della composizione, sottolineando l'indifferenza religiosa come la più grande colpa di degrado morale e spirituale dello stato della Chiesa e della società; mentre i religiosi ed i fedeli sono lontani da Dio, "o diabo tem o poboo de Christo em seu poder muyto sojugado".

Passiamo ora all'esame della composizione del Pianto della Chiesa redutta a mal stato di Jacopone da Todi. In questa lode, il francescano mette in scena la Santa Madre Chiesa lamentando la corruzione dei suoi figli:

Piagne la Ecclesia, piagne e dolura: senza fortuna di pessimo stato.

O nobilissima madre, che piangi?

Mustre Che senti dolur molto magni: narra me 'l modo perché tanto lagni,

che sì duro pianto fai esmesurato?

Figlio, eo sì piango, ché m’ aio anvito:

Veio me morto pate e marito;

figli, frategli, neputi ho smarrito,

onne mio amico è preso e legato.

So circundata da figli bastardi:

Em omne mia pugna se mustra codardi;

Li miei legitimi, spade né dardi, 
lo lor coraio non era mutato.

Li miei legitimi era'n concorda:

veio i bastardi pin de discórdia;

la gente enfedele me chiama la lorda

per lo rio essempio che ò semenato.

Veio esbannita la povertate

nullo è che cure si no 'n degnetate;

li miei legitimi em asperetate,

tutto lo monno ro fo conculcato.

Auro ed argento ò rebannito,

fatt'ònemici con lor gran convito,

omne buon uso da loro è fugito:

donne 'l mio pianto con granneeiulato.

O'so li? Patripieni di fide

Null'è che cure morir l'om me vide:

la tepedezam'ha preso edoccide,

el mio dolore non è corrottato.

O' so i profeti pin de speranza?

Null'è che cure en miavedovanza;

Presonzione presa ha baldanza,

Tutto lo monno po' lei s'è rizato.

O' so l' appostoli pin de fervore?

Null' è he cure en mio dolore:

Escito m' è scontra lo proprio amore,

e ia non veio che i sia contrastato.

O' so gli martiri pin de forteza?

Non è che cure em mia vedoveza:

escita m' è scontra l' ageveleza,

el mio fervore sì ha nichilato.

O' so i prelati iusti e fervente,

che la lor vita sanava la gente

Escit'è la pompa, grossura potente,

E sì Nobel ordene m' ha maculato. 
O' so i dotturi pin de prudenza?

Multi ne veio saliti en escienza;

ma la lor vita non m'ha convegnenza:

dato m’òcalci che 'l cor m' ò accorato.

O reliusi em temperamento, granne de vui avea piacemento; orvo cercano onne convento, pochine trovo en cui sia consolato.

O pace amara, co m’hai sì afflitta!

Mentre fui em pugna, sì stetti dritta; or lo reposo m'ha presa e sconfitta, el blando dracone sì m' ha venenato.

Null' è che venga al mio corrotto, en ciascun statosì m' è Cristo morto:

o vita mia, speranza e deporto, em onne coraio te veio affocato.

Così come la lode portoghese, questa composizione tratta la corruzione della Chiesa e la tiepidezza dei sacerdoti e della società.

Nonostante la conformità tra i temi, è possibile distinguere la cosmovisione di ogni autore.Infatti, paragonando le composizioni portoghesi con il testo iacoponico, possiamo notare "atteggiamenti" differenziati prima dello stesso tema - degradazione della società e istituzione religiosa.In questo senso, André Dias adotta il testo italiano come riferimento per la sua composizione, adottandolo come base e, da esso, aggiungendo o eliminando elementi secondo la sua visione del mondo.Mário Martins osserva:

[...] le versioni di Maestro André Dias si staccano dall'originale, hanno il valore di una creazione poetica che ci ricorda, qua e là, il nostro versetto modernistico. I versetti italiani, in questo caso, servivano come un punto d'appoggio per il decollo [...]. Anche quando non si stacca dalla lauda toscana, possiamo affermare Che il maestro André, più che tradurre in linguaggio portoghese sistemava portoghesemente, se ci permettete questo avverbio, e creava sullo stesso tema musicale e ideologico, una nuova poesia [...]. (MARTINS, 1951, pp. 25-26). 


\section{Laudes e cantigas espirituais de mestre André Dias: finalidade crítica e correlação com a obra de Jacoponi da Todi \\ Veridiana Skocic}

L'affermazione dello studioso attesta il rapporto tra le laudi portoghesi con gli scritti di Jacopone.Tuttavia, come possiamo osservare, questo dialogo si svolge soprattutto nella rielaborazione dei contenuti originali da cui viene costruita una nuova poesia. André Dias adatta il testo italiano secondo i requisiti del suo spirito di riforma, desideroso di criticare non solo lo stato della Chiesa, ma anche i laici.

Per quanto riguarda i due saggi analizzati, le analogie appaiono a partire dalla settima strofa, una volta che il maestro Andre "scarta" le prime istanze della composizione italiana. Sono i versi iniziali di ogni strofa successiva Che dimostrano la corrispondenza tra $\mathrm{i}$ testi o anche la traduzione letterale della composizione di Jacopone del vescovo di Megara:

\begin{tabular}{|c|c|}
\hline Ó so li patri pieni di fede? & E hu som os patriarchas plenos de fé e de creença \\
\hline O’ so i profeti pin de speranza? & e honde som os prophetas plenos de sabedoria \\
\hline O' so gli appostoli pin de fervore? & E hu som os apóstolos, preegadores da verdade \\
\hline O' so gli martiri pin de forteza? & E hu som os mártires plenos de fortaleza \\
\hline O' su i dotturi pin de prudenza? & E hu soom os doutores plenos de sabedoria \\
\hline $\begin{array}{l}\text { O pace amara, com'hai afflitta! } \\
\text { Mentre fui em pugna, } \\
\text { si stetti dritta; or lo reposo m'ha } \\
\text { presa e sconfitta, el brando dracone } \\
\text { si m'ha venenato }\end{array}$ & $\begin{array}{l}\text { Oo amara e triste paz e folgança...porquanto durou o } \\
\text { trabalho da egreja e da terra....esteve todo mundo, em } \\
\text { muyto grande devoçom, e agora no muyto grande } \\
\text { asossego, o diabo tem o poboo de Christo. }\end{array}$ \\
\hline
\end{tabular}

O sono i padri pieni di fede?

O sono i profeti pieni di speranza? E hu som os patriarchas plenos de fé e de creença

Osono gli apostoli pieni di fervore? e honde som os prophetas plenos de sabedoria

Osonoi martiri pieni di fortezza?

O sono i dottori pieni di prudenza?

O pace amara, come mi hai afflitta!

Mentre ero in battaglia, stetti dritta; ora il riposo mi ha preso e sconfitta,la spada e il drago E hu som os apóstolos, preegadores da verdade E hu som os mártires plenos de fortaleza E husoom os doutores plenos de sabedoria Oo amara e triste paz e folgança...porquanto durou o trabalho da egreja e da terra...esteve todo mundo, em muyto grande devoçom, e agora no muyto grande asossego, o diabo tem o poboo de Christo. così mi hanno avvelenato 
...Anche se ci sono qui e là passaggi tradotti alla lettera, André Dias adotta il testo iacoponico solo come base per la composizione, riformulando il contenuto originale, invertendo l'ordine delle strofe e dei versi. La sua critica è più completa; per il vescovo portoghese nessuno si salva. Tutti, religiosi e laici sono corrotti e l'inclusione di questo nuovo elemento, cioè il laico, è un fattore importante in relazione al testo italiano. Mentre Jacopone si limita all'immoralità dei religiosi: "So circundata da figli bastardi", Dias critica non solo il decadimento nell'interno della chiesa, ma anche i vizi della società (ottava strofa).

Viene evidenziato che il vescovo di Megara, oltre a inserire nuovi elementi in termini di contenuto, riconfigura in maniera strutturata la composizione originale, invertendo l'ordine delle strofe e cambiando il punto di vista. Nella composizione portoghese, il narratore in prima persona lancia una serie di domande senza risposte. Nella laude italiana, d'altra parte, questa relazione è stabilita, in primo luogo, in forma di dialogo - prima persona/interlocutore. Usando l'appello della personificazione, il soggetto poetico si rivolge alla Chiesa: "O nobelissima mamma, che piangi?" - Dalla seconda strofa sino alla fine c'è un cambiamento del punto di vista narrativo, perché è la Chiesa che si lamenta e che lancia le domande, passando dall'interlocutore al narratore in prima persona. Si osserva che, in entrambe le composizioni, l'assenza di risposte dimostra la disperazione e il pessimismo degli autori.

\section{Conclusione}

La conclusione, infine, è che tanto Jacopone come André Dias fanno una critica severa alle abitudini decadenti della Chiesa. Le loro lodi testimoniano l'oltraggio contro la corruzione dei religiosi e, nel caso di Dias, 
Laudes e cantigas espirituais de mestre André Dias: finalidade crítica e correlação com a obra de Jacoponi da Todi

Veridiana Skocic

anche contro la rovina morale che devasta la società di quel tempo. Il francescano, vivendo un processo in cui il clero, grazie alla ricchezza e alla conoscenza ottenute, è diventato corrotto e ambizioso; fa uso della personificazione (in armonia con l'allegoria medievale) attraverso la quale la Chiesa lamenta la corruzione dei suoi membri dimostrando sdegno per la decadenza della Curia romana; il vescovo portoghese, assistendo ad un momento crucialedi questo stato di degrado che colpisce anche la vita secolare, prende come riferimento il testo italiano, ricostruendolo a partiredalla sua esperienza, dall'ambiente e dalle sue percezioni.

La convergenza tra le lodi del vescovo di Megara e gli scritti del francescano Jacopone da Todi si trova nella critica di tiepidezza e degrado morale della Chiesa e della società. Dias e Jacopone attribuiscono una funzione critica, oltre che catechistica, alle lodi come abbiamo visto nelle composizioni Del pianto de la chiesa redutta a mal stato del frate italiano e Cantar quediram os desenparados. In questo modo, al di là dell'intenzione didattica, questo tipo di espressione religiosa sembra essere anche un efficace strumento di censura.

Infine, da quanto sopraddetto, è possibile dire che la lode (come parte integrante della letteratura religiosa), anche se basata su un quadro tematico meno esteso e una proposta più specifica della letteratura laica, è ugualmente diversificata a seconda che i suoi autori, nonostante abbiano lo stesso obiettivo e si limitino alla ricorrenza tematica, riescano a farla in modi distinti a partire dalla loro visione del mondo.

\section{Riferimenti bibliografici}

ALMEIDA Fortunato. História da Igreja em Portugal. 4 vols. Nova Edição. Porto: Livraria Civilização Editora, 1967-1971. 
Laudes e cantigas espirituais de mestre André Dias: finalidade crítica e correlação com a obra de Jacoponi da Todi

Veridiana Skocic

CALDERÓN, Manuel. O Livro de orações em prosa e verso vulgar com as laudas e cãtigasspirituaes de André Dias. In: Signum. Revista da Associação Brasileira de Estudos Medievais, 6, 2004, pp.45-88.

CANETTIERI, Paolo. "LaudediJacoponedaTodi." In: Letteraturaltaliana- dalle origini al Cinquecento. Torino: Einaudi, 1992.

DE SANCTIS, Francesco. Historia della literatura italiana. Buenos Aires: Editorial América, s/d, pp.32-33.

DIAS, André. Laudes e cantigas espirituais. Coligidas, anotadas e comentadas por Mário Martins. Lisboa: Oficinas Ramos, Afonso \& Moita, 1951.

GRENDI, Edoardo. "Le confraternite come fenomeno associativo e religioso", In Società, Chiesa e vita religiosa nell' "ancient régime', a cura di C. Russo. Napoli, 1976.

MARQUES, Antonio Henrique. A Sociedade Medieval Portuguesa. 5a. Ed. Lisboa: Livraria Sá da Costa, 1987.

MARTINS, Mario. A Bíblia na literatura medieval portuguesa. $1^{\mathrm{a}}$ ed. Lisboa: Livraria Bertrand Ed., 1979.

RICCI, Angelo. Umanità e popolo nella lauda del secolo XIII. Porto Alegre: Imprensa Universitária Editora, 1971.

ROMAG, Dagoberto. Compêndio de história da Igreja - Idade Média. $2^{\mathrm{a}}$ ed. Vol. II. Petrópolis: Vozes, 1950.

TODI, Jacoponi. Le laudi. A cura di Giovanni Ferri. Laterza, 1915.

VISALLI, Angelita. Cantando até que a morte nos salve: estudo sobre as laudas italianas dos séculos XIII e XIV. Tese de doutorado, USP, São Paulo, 2004. 
Laudes e cantigas espirituais de mestre André Dias: finalidade crítica e correlação com a obra de Jacoponi da Todi

Veridiana Skocic 